6. The small pneumothorax which sometimes remains after the healing of a cavity, as has been demonstrated by the roentgen ray, is negligible, since it disappears spontaneously in a short time.

Twenty of the operations were performed by myself, the remainder by various attending surgeons of the hospital, to whom I am greatly indebted for carrying out the suggestions which I believed essential to securing so low a mortality in the treatment of our empyema cases.

The fact that we were enabled to trace end-results in all but eight of our cases shows one of the many adrantages of an efficient hospital social service.

\section{THE TREATMENT OF MUCOUS COLITIS *}

W. A. BASTEDO, M.D.

Assistant Professor of Clinical Medicine, Columbia University College of Physicians and Surgeons NEW YORK

The name mucous colitis (myxomembranous colitis) stands for a poorly understood clinical entity, and I shall therefore preface my remarks by a brief review of what is implied in the condition as I understand it. It may be defined as a condition characterized by the formation in the large bowel of great quantities of abnormal mucus. It should not be termed "colica mucosa" or "mucous colic," for colic occurs in only a small proportion of the cases. The disease may occur at any period of life from infancy to old age, and contrary to the prevailing belief, is encountered in males quite commonly as well as in females. It is probably hereditary to some degree, as I have found it repeatedly in parent and child, when I have usually noted in the roentgenogram the presence in each of the same type of redundant or looped colon, often with ptosis.

Though mucous colitis is of frequent occurrence, yet it appears to be infrequently recognized. The importance of its recognition is evident if we realize that, although at times the condition of the bowel may be merely incidental to more important lesions, it is often most prominently associated with serious states of physical debility or of mental instability. I would not say that it is a cause of these, but it does strikingly increase their manifestations, and its cure is not infrequently accompanied by their disappearance.

In severe mucous colitis the three outstanding manifestations are: abdominal pain, intestinal putrefactive toxemia and neurotic manifestations. Whether the patients are classed as suffering from colitis, or from neurasthenia or psychoneurosis, or from autointoxication, the mucous colitis must be treated or the patient will not be relieved of the other manifestations.

Usually, the patients seek a physician, not because of the colitis, but rather for persistent constipation, abdominal pain or distress, lack of physical energy, obstinate or recurrent headache, failure of physical or intellectual vigor, mental depression or complete nervous breakdown. Though they may have noticed strings of mucus in the stools, the patients commonly do not mention this unless interrogated. Hence, in a patient showing nervousness, depression of spirit, and lack of the usual forcefulness, it is well to search for a possible mucous colitis.

* Read before the Alumni Association of the University of Buffalo Department of Medicine, June 21, 1919, and the Medical Society of Atlantic County. N. J.. Nov. 14, 1919.

\section{DIAGNOSIS}

As there is no distinguishing factor other than the mucus, the diagnosis rests on: (1) the observation by the patient of the passage of mucus, either as strings accompanying the feces or as a complete mucous stool (2) the finding of the characteristic mucus in a stool submitted; (3) the detection of the characteristic mucus after a test dose of castor oil or a test colon irrigation, or (4) the observation through a sigmoidoscope of a dry mucous membrane in the upper rectum to which are clinging the heavy, tenacious sheets of mucus. If mucous colitis is sought for only when there is colic, many of the most important cases will be overlooked.

Mucus.-This is tenacious and appears mostly in yellow, brown or sometimes black jelly-like masses, or in more or less desiccated plaques, strings, ropes, or scablike membranes. The strings may be many inches in length, and on being teased out in water prove to be casts of the bowel, or broad ribbons. Patients sometimes mistake them for intestinal worms. The mucus adheres with great tenacity to the wall of the bowel, so that when encountered postmortem it is removed with difficulty, even with forceps; von Noorden reports that he was unable to drive it off with a strong stream of water from a hydrant. Hence, it is not uncommon to find blood spots on the expelled mucus, as if it had been torn away like a scab. As a rule, the mucus bears only a few leukocytes and is without fibrin. In some of the persistent cases I have frequently noted about the mucus an odor that is entirely different from that of the stools, slightly suggestive of dead fish just thrown up on the beach and before they have become offensively putrefactive.

Besides mucus there are occasionally found quantities of blackish or brownish, gritty, irritating intestinal sand. Some authors report the frequent presence of blood in the stools (Mummery found it in 60 per cent. of cases), but after thousands of examinations I can report that this is not usual, and occurs mostly when the scablike membranes come away. It is regularly present in cancer cases, and frequently with polyposis; but in these cases the mucous colitis is not the important feature.

Pathology.-The changes are indefinite; the colon sometimes shows very slight alterations or even rarely no recognizable lesion, but usually the mucous membrane is more or less danaged. In some of the colons removed at operation the mucous membrane of the cecum and ascending colon has revealed a universally distributed area of inflammation, and the whole wall of this part of the intestine has shown atony, dilatation and poor nutrition. In some cases Keith has demonstrated absence of the normal nerve elements of Auerbach's plexus with consequent cecal and colonic stasis. The colon is often redundant or looped, and there is sometimes laxity of the abdominopelvic walls with ptosis of the transverse colon and of one or both flexures, with perhaps. a movable cecum. In the persistently toxic cases, pyorrhea alveolaris and gastric achylia are not uncommon findings. Rarely cancer of the colon, polyposis, diverticulosis or some other localized lesion may underlie the condition. Not infrequently the descending colon is found in a spastic state.

Pain.-In a large proportion of the cases a certain amount of discomfort is manifested at some stage of the disease. It may range in intensity from nothing more than a little soreness to paroxysms of the most distressing colic; its location may be variable or con- 
stant, and in the latter event it has not infrequently led to a futile operation for some supposedly serious abdominal or pelvic lesion. At times, the whole colon, or a part of it, is found to be tender. The colic is the direct result of the attempt by the bowel to expel longretained and clinging mucus; persistence of the attacks may be taken as an indication that not all the mucus has been liberated. The mucus, acting as an irritant, induces spasmodic contraction of a portion of the colon, and the pain is due to tension in the distended portion above this. The spasms do not produce the painful manifestations, for, as Hurst has shown, bowel pain comes from distention, not from contraction. The pain ceases after the reestablishment of the peristaltic reflex with dilatation below and contraction above.

It is to be remembered that pain of significant degree is lacking in some of the cases, and in many is absent for considerable periods. Yet even during the painless periods the symptoms are more manifest when the mucus is retained, and become less so following its expulsion.

Constipation.-In some cases several stools a day are passed, yet constipation exists, for the cecal cess- pool seems never to be emptied. Constipation is not necessarily a condition of infrequent defecation, but is rather one of insufficient or retarded defecation (Hurst) ; colon irrigations have shown that there may be considerable cecal retention of putrefactive contents, though the bowels seem freely open.

Intestinal Putrefactive Toxemia. - Without doubt, the neurotic manifestations and the condition of mental and physical fatigability may in many instances be traced directly to a chronic toxemia caused by the absorption of harmful chemical substances formed in the bowel. And it is probable not only that the constipated bowel of mucous colitis favors bacterial proteolysis, but also that the damaged mucous membrane promotes the absorption of deleterious material. The subject of intestinal toxemia is too large to be dealt with here; it is important to remember, however, that quite often the treatment of mucous colitis involves extensive consideration of the associated toxemia. There may be mucous colitis without putrefaction of the colon contents, or there may be putrefaction of the contents without colitis, but the two conditions are often associated.

\section{COMPLICATIONS}

Occasionally we encounter bronchial asthma, urticaria, angioneurotic edema, or erythema, complications which suggest either protein sensitization as a restalt of absorption of minute amounts of unchanged protein through the diseased bowel wall, or the possible absorption of some chemical formed in the bowel. For instance, histamin is at times a product of intestinal putrefaction, and when absorbed in sufficient amounts is capable of producirig asthma, urticaria and angioneurotic edema.

A phenomenon, usually associated with gastric atony and observed by me in a few cases, is tetany.

Nervous or Psychic Manifestations.--Many of these patients, though not by any means all, have a variety of nervous symptoms. These may range all the way from a simple impressionability and hypersensitiveness in insignificant matters to severe nervous breakdown or such mental instability, lack of self-control and irresponsibility of action as to bring the patient to the borderline of insanity. Indeed, it is often quite impossible to decide whether the mucous colitis or the psychoneurosis is the primary condition. Conspicuous in many are lack of self-confidence, absence of initiative, autosuggestibility, spells of discouragement, and fatigability, both mental and physical. Interesting are the phobias, such as the dread of visitors, of riding in a train, and of being alone at home or in the city streets. The colitis may begin with, or be aggravated by, a nervous strain, such as worry over money matters, over a wayward child, over a husband who drinks or over some skeleton in the closet, or by the assumption of social or business responsibilities that seem utterly beyond the patient's powers.

In a person of dominant type this onset of inability to cope with the daily responsibilities makes for discouragement, mental depression and fits of dejection. I have known strong men, physicians, lawyers, heads of great business, to be overcome by their feelings of helplessness and to burst into tears in my office. I have seen the president of a great concern weep while saying, "Doctor, I cannot face my directors at tomorrow's meeting." I have heard a medical school professor cry, "It's of no use my lecturing any more, I cannot hold the boys, I have lost my power." One patient, a strong man who had repeatedly put up a 100 pound dumb-bell as many as nine times, in the most doleful manner deplored the fact that he could not then get it up once. And each of these men, as the mucous colitis yielded to treatment, regained his old dominance and power. Sometimes such patients have slipped back again, and have been found to have a new access of the colitis.

What is the relation of the bowel trouble to the nervous system? Perhaps in some of the cases there is the same underlying cause for both the intestinal and the nervous symptoms. Among neurologists it is customary, however, to consider that neurasthenia or a psychoneurosis requires two conditions for its production, namely, an underlying mental deficiency, and a provocative factor the most pronounced of which is fatigue. Yet perhaps the underlying mental deficiency is not such an absolute requirement, for Féré says that "fatigue often provokes ideas of negation, persecution and disparagement," whereas Dubois remarks that "exaggerated fatigue may induce neurasthenic states in the best balanced individual." If, then, fatigue is the great provocative factor, may it not be that mucous colitis is one of the important producers of fatigue, either of itself or through the production of toxic substances? Certainly fatigue and fatigability are among the most striking accompaniments of mucous colitis, the patients being readily brought to a state of exhaustion by serious reading, by visitors, by responsibilities of any kind, or by physical exertion. In fact, they may feel fatigued even though they do nothing. So it is my belief that one of the important causes of these neurasthenic or psychoneurotic conditions is the fatigue or the fatigability which accompanies a mucous colitis.

Furthermore, as mucous colitis may induce fatigue or fatigability, so fatigue from other causes may increàse the colitis. Therefore, one of my rules for such patients is "Never get tired, physically, mentally or emotionally."

\section{PROGNOSIS}

I have seen many patients in serious nervous states, who, after the cure of the bowel trouble, have ceased to be more than normally nervous; and because of the possibility or probability of such a happy result, I lay stress on the treatment of the bowel. I believe that 
in a large number of these cases a cure is possible, but usually only after persistent treatment for a long time. And I believe that continued or repeated severity in the symptoms is often the result of neglect by the patient or the physician. The classification of the patient as a "neurasthenic" makes the outlook hopeless, for the physician then does nothing. The height of absurdity was reached by a speaker at a recent meeting of the Pennsylvania State Medical Association, when he said that "the prognosis is absolutely hopeless, the treatment is nil, and the sole prophylaxis would have been to sterilize the grandfather."

\section{TREATMENT}

In determining the method of treatment, two facts stand out prominently: (1) that retained mucus is harmful mucus, and (2) that the cure requires a long course of treatment. I am wont to tell my patients that a year is the minimum time in which a cure can be effected. The treatment is directed at the prevention of the accumulation of mucus, and at the removal of the associated conditions, such as colic, constipation, intestinal toxemia, disturbed gastric conditions, bad mental states and depressed general health. We shall take up, first, the treatment of the condition when there is no colic, or after the attacks of colic, and then the treatment of attacks of colic.

I. Treatment in Cases Without Colic or After the Attacks of Colic. - This is designed $(a)$ to prevent accumulation of mucus; $(b)$ to overcome constipation and intestinal toxemia, and $(c)$ to improve the nervous and general health.

(a) To overcome accumulation of mucus, one of the best measures is a weekly purge with castor oil, or perhaps calomel and salts, or a purgative blue mass or calomel pill. This may be supplemented by colon irrigations every day or two for a week, and then every three or four days for two to several weeks longer, or once a week for longer periods of time. For this colonic lavage the patient should be on the left side for the first gallon of water in order to clean out the lower colon, and then on the back for the rest of the irrigation in order to enable the fluid to reach the cecum. Sometime preceding the lavage the bowels should be emptied, if necessary by an enema, in order to avoid carrying feces back to the cecum and to avoicl starting up by the lavage the defecation reflexes which will prevent the passage of the water into the upper colon. The liquid used should be rather hot, and I regularly employ tap water, though occasionally sodium bicarbonate solution, a dran to the pint (sodium bicarbonate is changed to carbonate by heat). Physiologic sodium chlorid solution is not employed, because it makes the patient thirsty. The amount required is usually from 12 to 24 quarts, and it should be given slowly, with the reservoir about two feet above the patient. Frequently the putrefactive cecal contents are reached only after 4 or 5 gallons have been employed. If the irrigation does not bring out the mucus, this may be expelled one or several hours later. I always consider an irrigation a failure if no water is retained to be evacuated after the irrigation is finished, or if the water returns clear throughout; obviously, in such cases the liquid has failed to get past the spastic descending colon or sigmoid. Recently transduodenal lavage with 4 per cent. sodium sulphate solution has been employed with reported success. I have not used it for this purpose.
It is to be noted that when the mucus is readily discharged from the bowel the patient is safe; danger threatens when the mucus stays in and is not readily loosened and expelled. Many of the patients learn this fact, and when the mucus suddenly ceases to appear they will use every endeavor to clear out the bowel in order to avoid a renewal of their symptoms. Sometimes a cathartic taken the same night as an irrigation that seems unsuccessful may bring away great quantities of mucus that has apparently been softened by the irrigation water.

(b) The treatment of the constipation and intestinal toxemia is that of any form of constipation. There should be insistence on regularity of bowel movements, but restriction of the use of enemas and colon irrigations. I have seen patients who had come to think that any abnormal sensation could be removed only by enema or irrigation, and who had acquired the habit of using these several times a day. The diet must be ample, for in case of lack of food residue it will be necessary to keep increasing the laxatives. A diet containing bran, fruits and coarse vegetables may be effective, but in the beginning this is usually more harmful to the bowel than a mild tonic laxative. Exercise does not help to overcome constipation, except as it may result in increased appetite. There have been many athletes among my constipated patients. If there is ptosis of the abdominal viscera with laxity of the abdominopelvic walls (and these are exceedingly common), the patient should wear an inelastic binder for mechanical support, and should be put on exercise to help to strengthen the abdominal muscles. The patient should drink freely of water.

The best of laxatives is usually a softening and bulk-producing agent, such as liquid petrolatum, cascara agar, phenolphthalein agar, or small doses of milk of magnesia or salts. But these are not always effective and frequently must be supplemented by one of the tonic laxatives, cascara, rhubarb, aloes, senna, or senna with sulphur in the form of compound licorice powder. A measure of occasional value is the retention in the rectum over night of from 4 to 16 ounces of olive or cottonseed oil. In these chronic cases the drastic cathartics may be successful in getting out mucus, but should be employed only seldom. If such measures as these do not overcome the stasis and the toxemia, the question of surgery should be seriously considered.

Hemorrhoids are a bad complication, as they prevent the use of irrigations, enemas or such strong cathartics as castor oil. They may be treated by the nightly instillation into the rectum, by means of a soft rubber ear syringe, of 60 c.c. of warm olive or cottonseed oil to be retained over night.

(c) Improvement in the nervous and general health. The diet may have to be modified according to the conditions of the stomach and upper bowel (achylia, hyperchlorhydria, gastric atony, etc.), and to overlook these is to fail in the treatment; but, in general, at the outset the diet should be of the bland lactofarinaceous type. Later there may be a gradual transition to a coarser type with sufficient vegetable and fruit. But there should be at all times limitation in the amount of readily putrefactive proteins, as found in animal flesh, eggs, beans, peas and lentils, these being replaced, if possible, by much milk in the dietary. Of these proteins, in my experience, those of chicken and egg are most prone to produce putrefaction; and peas and beans are more harmful when dried than when 
green. The coarse diet at the outset, as advocated by von Noorden, is likely to give rise to gastric disturbances, particularly flatulence, and it seems quite rational to assume that an excessive quantity of coarse, indigestible, fermenting food will do more harm to some of these damaged colons than any mild laxative drug. But in all cases the diet should be ample and its quantity insisted on, for these patients readily acquire the habit of undereating because of a suspicion that this, that, or the other article of food does not agree with them.

If there is any definite surgical condition in the abdomen, such as appendicitis, cholelithiasis, adhesions, bands or pelvic disturbances, it should be overcome. In persistent cases, some surgeons operate on the bowel itself: (1) For cleansing and medicating the colon; as by appendicostomy or cecostomy, for the purpose of permitting daily irrigations through the whole colon. But these must be continued for from six months to two years (Mummery), and the procedure is not, in my opinion, a valuable one. (2) For overcoming stasis or for the removal of diseased portions of the bowel, by plication of the cecum, ileosigmoidostomy, cecosigmoidostomy, partial colectomy and complete colectomy. The indications for radical surgery and the choice of surgical procedure I shall not attempt to discuss here.

Occupation, recreation and rest in proper proportion should be advised. The patients must not be allowed to coddle themselves. They should get up before breakfast and not lie in bed in the morning; they should recline when possible, at a later period in the day, perhaps best for one or two hours after the midday meal, and they should retire early. They should give less time than usual to social or business responsibilities or give these up entirely, and they should not receive too many visitors. They should avoid adding unnecessary responsibilities to the necessary ones; for example, in a schoolteacher, the taking of extra college courses on Saturday, and on Sunday the teaching of Sunday School; in a business man the devotion of his evenings to club committees or to study. They should not undertake reading of too serious a character. It is of paramount importance that, for the time being, patients give up their ambitions, whether social or otherwise. They must, however, have some recreation, preferably golf, horseback riding, bathing or other outdoor exercise, or attendance at games in the open air.

The great rule is that patients must never get unduly fatigued either physically, mentally or emotionally. I explain to them at the outset that they are temporarily handicapped, and therefore cannot do as much as normal people. On the other hand, I do not allow them to shirk all responsibility, but try to get them to do as much as lies well within their powers, encouraging them to attempt more and more till their full powers are restored. Some women with money take matters too easily and are prone to become chronic invalids, self-centered, hypochondriac, "hipped" on themselves, and leading useless lives. They go from one physician to another, or to sanatoriums, read numerous books about their disease and its treatment, and refuse to permit their ailment to be forgotten for a moment. These patients should be induced to avoid medical books and constant examination of their stools, and in every way they should be encouraged to use their faculties and their muscles in a sensible manner, neither too much nor too little. A wise nurse is a great help in managing the daily life of the female indolent patient.

General hygienic measures, such as cold spinal douches, or alternating cold and hot douches, cold morning baths, cold rubbings up and down the spine, calisthenics, and general massage, with very gentle abdominal massage, are of distinct advantage. A help in the treatment is a change of scene to get away from oversolicitous or nagging friends, or from the wear and tear of home or business. He was a physician of deep perception who replied to the inquiries of the overanxious wife: "Madam, your husband needs a rest. One of you must take a vacation." A visit to one of the spas may furnish rest and recreation away from business and friends and amid pleasant surroundings, with the advantages of hydrotherapy and perhaps the daily ingestion of laxative waters. It is well to remember that nervous people do not sleep or rest well in high, mountainous regions.

The use of tobacco and alcohol, and usually also of coffee, should be prohibited.

Bromids, in doses of from 1 to $2 \mathrm{gm}$. (15 to 30 grains) once or twice a day are indicated for a short time during the most nervous periods.

In severe cases the patient should be put to bed in charge of a competent nurse. It is bad practice, because of the effect of loneliness, to keep the patient in bed without an attendant.

II. Treatment of Attacks of Colic.-This resolves itself into measures (1) to relieve pain and neurotic symptoms, and ( 2 ) to promote evacuation of the nucus. These patients feel pain keenly and may writhe in the attacks. of colic, and they may even throw themselves out of bed.

1. The pain and neurotic symptoms may be relieved by rest in bed, a large dose of bromid, 2 to $4 \mathrm{gm}$. $(1 / 2$ to 1 dram) by mouth, a hypodermic of atropin sulphate, $0.001 \mathrm{gm}$. (1/65 grain), with codein phospluate, $0.03 \mathrm{gm}$. ( $1 / 2$ grain), and hot applications to the abdomen in the form of a hot water bag, electric pad, poultice or stupe, or a hot bath. On account of habit formation in neurotic subjects, morphin should not ordinarily be employed; but when the recurrence is not frequent and the attack is very severe, morphin may be the best remedy. To relieve the colic the best single drug is atropin; it is of no use for the cure of the colitis.

2. To promote the evacuation of mucus one may use (a) a large dose of castor oil by mouth. This acts by making vigorous peristalsis, which, coming from above the mucus, tends to separate this from above downward and to carry it onward, while at the same time it abolishes the spasmodic obstruction through the peristaltic reflex which produces dilatation below and contraction above. (b) Colon irrigations with tap water or a solution of sodium bicarbonate, a dram to the pint, should be given warm and at low pressure ( 2 feet). On account of the spasticity of the descending colon it may be impossible to get the liquid up into the colon at first, but persistence and gentleness may result in success. Irritants, such as silver nitrate, should not be employed in the already highly sensitive colon.

Often the combination of castor oil by mouth, codein and atropin hypodermically, and colonic lavage will be followed by relief and sleep. But if the attack is very severe and persistent, and especially if the irrigation is unsuccessful, it is a good plan to put the patient in the knee-chest position and to inject slowly into the 
colon from one-half to one pint of warm olive oil or cottonseed oil, to be retained over night or as long as possible, a towel being placed over the anns and a rubber sheet on the bed as protection in case of leakage. This injection is often followed by the passage, a few hours later, of the oil and an abundance of mucus, with disappearance of the colic and no recurrence for a long time, if at all.

57 West Fifty-Eighth Street.

\section{METHODS OF ADMINISTERING SAI.INE AND OTHER SOLUTIONS TO INFANTS AND CHILDREN *}

\author{
JOHN AIKMAN, M.D. \\ ROCHESTER, N. Y
}

The administration of physiologic sodium chloricl and other solutions to replace fluids lost from the body is a valuable method of carrying certain cases over critical periods. Loss of fluid is more serious in children because of the vomiting that so often accompanies grave illness, and because of the difficulty of giving water by mouth. A relative acidosis may easily result, which in a few hours may greatly increase the gravity of the attack. The reduction of fluid is marked in cases with a history of numerous watery stools, characterized by a rapid loss of weight, hollow eyes, and drawn, pinched expression of the facies. It is my object here to present an outline of the several methods of administering fluids, and to discuss their relative merits.

The method of greatest service is that which will permit the introduction and retention of large amounts of fluid with the greatest ease to the operator and with the least danger and discomfort to the patient.

\section{OUTLINE OF METIIODS}

1. Administration of Fluid by Mouth.-If a child can take and retain sufficient quantities by mouth, it is obvious that no other method is necessary, except for special indications, such as a great loss of blood as the result of hemorrhage.

2. Rectal Administration. - The introduction of fluids by rectum, either through enemas, return flow tubes, or by the drip method has been employed for some years with satisfactory results. If the fluid is properly introduced, there is no doubt that considerable absorption takes place. The method is objectionable in children, because of factors not encountered in adults. Unless the child is prostrated, he is apt to be restless, making the retention of the tubes very difficult. It takes considerable time to introduce the necessary amount of fluid, and the child is more or less disturbed thereby, which interferes with his rest. The fluid is apt to be promptly expelled, especially in diarrhea, and there is always some doubt as to the amount which has been absorbed. However, a great variety of fluids may be given by this method, and there is no special danger attached to the procedure.

3. Hypodermoclysis.-In children, especially very small children, small amounts of fluid can be quickly given by this method through a Luer syringe and an intravenous needle, or it can be given by gravity. We have often given fluid in several different areas at

* Read before the Rochester Pathological Society, Nov. 19, 1919. the same time. The procedure is very painful and causes restlessness, especially in older children. The amount that can be given is quite limited. While absorption is slow it is sure. It is safest to use physiologic sodium chlorid solution, although I once used a fresh solution of 1 per cent. sodium bicarbonate in two children over 5 years of age who were desperately ill with ileocolitis accompanied by vomiting and very frequent stools. No trouble resulted from repeated injection of fluid, and both children made an uneventful recovery; there is always danger that solutions of sodium bicarbonate will change in character and cause a slough. These cases were seen several years ago; under similar conditions today I would give physiologic sodium chlorid solution by another method that would better answer the indications and cause much less pain.

4. Intravenous Injection.-It is not necessary to mention all the fluids that may be given by this method. It is estimated that an amount equal to one sixtieth of the body weight can be introduced through the veins, but much more can be used if the loss of fluid has been extreme. Excretion of the fluid begins at once; if prolonged results are to be obtained, it is often necessary to give repeated infusions. The limited amount that may be given and the difficulty of entering the vein of a small child are the chief objections to this method. With proper technic the method is safe, but it will be acknowledged by any one who has tried it that it is difficult to make an intravenous injection on a small, active infant, especially into the veins at the elbow. In order to overcome this difficulty, other methods have been devised.

(a) Intrasinus Injection: In an infant with open fontanel this offers the best means of introducing fluid into the blood stream. The method was first studied by Tobler and was introduced in this country only a few years ago by Helmholz. By this technic, the fluid can be injected through the anterior fontanel directly into the superior longitudinal sinus. As the sinus lies from 2 to 5 millimeters from the skin, it can be easily entered if the fontanel is not closed; at the posterior angle of the fontanel the sinus is wider and deeper. The child is held prone on the table by an assistant, while the needle is introduced in the median line just in front of the posterior angle. If the child is quiet, it is very easy to withdraw blood or to introduce fluid; by means of a Luer syringe, rubber tubing and a threeway cock any amount of fluid can be given without removing the syringe. The needle should be short, and the long point usually found on intravenous needles should be filed away. If a glass syringe is attached before introduction of the needle, constant suction may be maintained for the purpose of discerning when the sinus is entered. If negative pressure is. not produced, blood will not flow so quickly, while the operator may push the needle through the inferior wall of the sinus, blood flowing only when the needle is withdrawn. This accident may also be avoided if the needle be introduced at an angle, directed backward.

Any solution adapted to intravenous administration can be given in this way; with physiologic sodium chlorid, glucose and other mild solutions there is practically no danger. It is also an excellent method for transfusion of citrated whole blood in infants.

In cases with a closed fontanel, the external jugular vein can often be used successfully.

(b) Injection in the Femoral Vein: This method is practicel in this city by several physicians working 